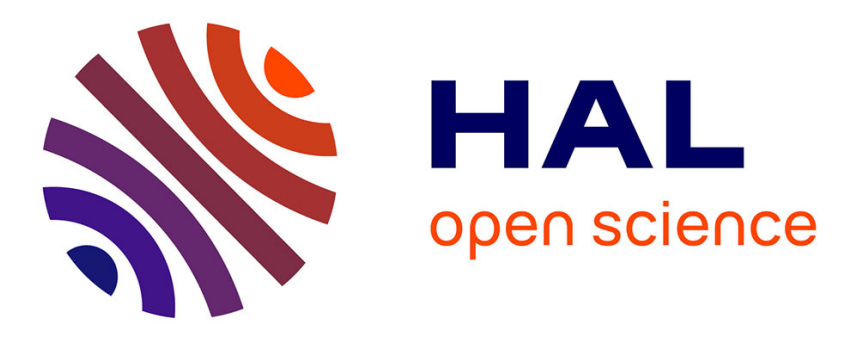

\title{
Real-time 3-D Spectral Doppler Analysis with a Sparse Spiral Array
}

Alessandro Ramalli, Enrico Boni, Claudio Giangrossi, Paolo Mattesini, Alessandro Dallai, Herve Liebgott, Piero Tortoli

\section{To cite this version:}

Alessandro Ramalli, Enrico Boni, Claudio Giangrossi, Paolo Mattesini, Alessandro Dallai, et al.. Realtime 3-D Spectral Doppler Analysis with a Sparse Spiral Array. IEEE Transactions on Ultrasonics, Ferroelectrics and Frequency Control, 2021, pp.1-1. 10.1109/TUFFC.2021.3051628 . hal-03116046

\section{HAL Id: hal-03116046 \\ https://hal.science/hal-03116046}

Submitted on 20 Jan 2021

HAL is a multi-disciplinary open access archive for the deposit and dissemination of scientific research documents, whether they are published or not. The documents may come from teaching and research institutions in France or abroad, or from public or private research centers.
L'archive ouverte pluridisciplinaire HAL, est destinée au dépôt et à la diffusion de documents scientifiques de niveau recherche, publiés ou non, émanant des établissements d'enseignement et de recherche français ou étrangers, des laboratoires publics ou privés. 


\title{
Real-time 3-D Spectral Doppler Analysis with a Sparse Spiral Array
}

\author{
Alessandro Ramalli, Enrico Boni, Claudio Giangrossi, Paolo Mattesini, Alessandro Dallai, \\ Hervé Liebgott, Piero Tortoli
}

\begin{abstract}
D sparse arrays may push the development of lowcost 3-D systems, not needing to control thousands of elements by expensive application-specific integrated circuits. However, there is still some concern about their suitability in applications, such as Doppler investigation, which inherently involve poor signal-tonoise ratios (SNRs).

In this paper, a novel real-time 3-D pulsed-wave Doppler system, based on a 256-element 2-D spiral array, is presented. Coded transmission and matched filtering were implemented to improve the system SNR. Standard sonograms as well as multigate spectral Doppler (MSD) profiles, along lines that can be arbitrarily located in different planes, are presented. The performance of the system was assessed quantitatively on experimental data obtained from a straight tube flow phantom. An SNR increase of $11.4 \mathrm{~dB}$ was measured by transmitting linear chirps instead of standard sinusoidal bursts. For a qualitative assessment of the system performance in more realistic conditions, an anthropomorphic phantom of the carotid arteries was used. Finally, real-time B-mode and MSD images were obtained from healthy volunteers.
\end{abstract}

Index Terms-Three-dimensional ultrasound, sparse arrays, pulsed wave Doppler, real-time system, coded transmission, pulse compression, ULA-OP.

\section{INTRODUCTION}

Over the last four decades, ultrasound spectral Doppler has been the method preferred by medical doctors to quantitatively assess blood flow [1]-[6]. Although traditionally limited to the detection of velocities within a single sample volume, spectral analysis has also been successfully extended to multiple sample volumes aligned along a scan line (multigate spectral Doppler - MSD), for vascular investigations [7].

In recent years, massive research efforts and technological developments have brought two breakthroughs: volumetric (3D) imaging [8]-[11] and vector Doppler [12]-[23]. Although medical doctors always need some time to fully accept new display modalities, both methodologies are mature for implementation in ultrasound machines. Furthermore, the volumetric morphological view (e.g., of plaques) could finally avoid the unfair assumptions that are often done when using pulsed-wave (PW) Doppler with 2-D scanners [24]. The possible extension of spectral-analysis-based velocity investigation to all points within an entire volume is, thus, of undoubtful clinical interest. Indeed, the support of a 3-D morphological view may facilitate the optimal probe

A. Ramalli, E.Boni, C. Giangrossi, P. Mattesini, A. Dallai, and P. Tortoli are with the Department of Information Engineering, University of Florence, 50139 Florence, Italy. positioning and the rapid identification of regions in which spectral analysis may provide valuable flow information.

3 -D imaging is currently possible only through a few highend machines based on expensive 2-D transducer arrays with thousands of elements controlled by application-specific integrated circuits (ASICs) [25]-[28], which relax the system requirements in terms of independent channels. A cheaper alternative is represented by sparse arrays [29], [30], which can be designed to have the number of active elements equal to the number of channels available in a companion scanner. In general, the design approaches for sparse arrays can be split into two groups: deterministic [30]-[32] and nondeterministic [33][36]. Among the latter ones, in [37], [38], an algorithm based on simulated annealing and broadband simulations was developed. It was shown able to find the set of active elements, which optimize the beam pattern, by considering both the pressure field behavior at different depths and some fabrication constraints. A deterministic approach was followed in [39], in which the array layout was based on the Fermat's spiral, which guarantees a uniform performance for focused beams steered over a wide range of angles. Moreover, spatial density tapering of elements was proposed to decrease the sidelobe level while increasing the probe sensitivity.

A recent study compared the PW Doppler performance of a full 1024-element gridded array and a sparse 2-D array, which was obtained by selecting 256 elements out of the full array [38]. The two array configurations were used to transmit focused beams into different directions and to Doppler process the echo-data off-line [40]. The results have demonstrated that the use of the sparse array only limits the signal-to-noise ratio (SNR), but neither the Doppler spectrum shape nor the detected mean frequency are affected by sparsity.

In this paper, a novel 3-D Doppler system based on a 256element spiral array is presented and shown able to provide, in real-time, multigate spectral Doppler profiles along lines that can be arbitrarily located in a volume. The implementation of coded transmission (TX), together with matched filtering in reception (RX), is also described, and shown capable of partially overcoming the SNR limitation of sparse arrays.

The paper is organized as follows: Section II introduces the hardware used in this study, including a 2-D array probe and the ULA-OP 256 research scanner. The methods used to evaluate the system performance are also reported. The experimental results are described in Sec. III and discussed in Sec. IV.

H. Liebgott is with the Université de Lyon, INSA-Lyon, Université Claude Bernard Lyon 1, UJM-Saint Etienne, CNRS, Inserm, CREATIS UMR 5220, U1206, Villeurbanne, France. 


\section{MEthodS}

\section{A. Real-time 3D Doppler system}

\section{1) General overview}

In this work, the ULA-OP 256 research system [41] was coupled to a special version of the Vermon matrix array probe (Vermon S.A., Tours, France). The ULA-OP 256 is a hardwarebased open scanner [42], which allows implementing custom processing methods by programming computing devices such as field-programmable gate arrays (FPGAs) and Digital Signal Processors (DSPs). As shown in Fig. 1, the special version of the 2-D probe was obtained by sparsely selecting 256 elements out of the original array. It consists of 32 (x-axis) by 35 (y-axis) elements $(3.7 \mathrm{MHz}, 300 \mu \mathrm{m}$ pitch, $70 \%$ bandwidth), but on the $y$-direction every ninth row is not connected. The total number of addressable elements is thus 1024. Although not easily recognizable, the selection matches, as much as possible, the layout of a 2-D spiral whose density is tapered with a Tukey window [43], [44]. Each selected element was paired to one channel of the ULA-OP 256 scanner so that it could be individually controlled both in transmission (TX) and in reception (RX).

The scanner software and firmware were modified to permit navigation in the volume of interest. B-mode images, taken on planes defined by a programmable rotational angle, are simultaneously displayed to locate non-isoplanar Doppler lines over the vessels of interest.

In the next paragraphs, the current implementation of the system is detailed.

\section{2) Transmission}

For both B-mode and Doppler imaging, the ULA-OP 256 is configured to transmit focused beams with programmable focal depth. No apodization is applied in TX since the spiral layout implemented density tapering. For each Doppler line, either tone bursts or linear frequency modulated (chirp) signals are used in TX to drive each active element with the same $35 \mathrm{~V}$

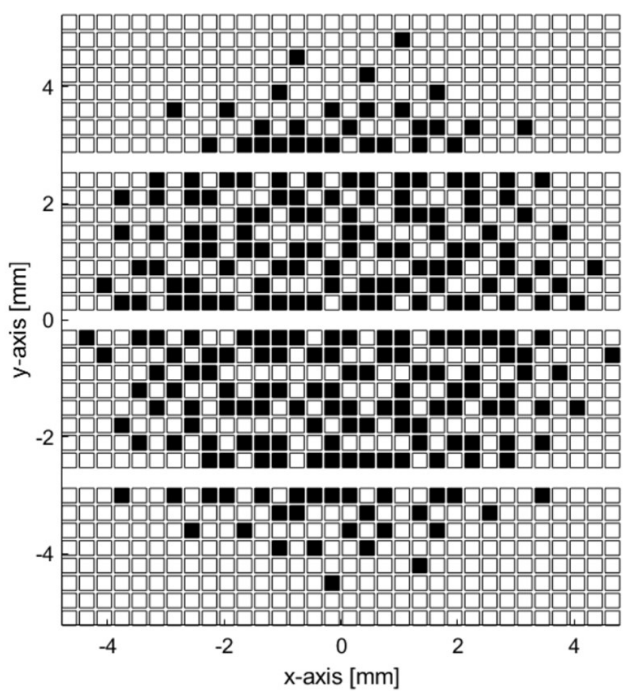

Fig. 1 Layout of the special version of the Vermon matrix array probe. Each selected element (black) was paired to one channel of the ULA-OP 256 scanner. peak amplitude (see Fig. 2). The chirp is $5 \mu$ s long, linearly spans a band of $3 \mathrm{MHz}$ centered at $3.7 \mathrm{MHz}$, and is tapered with a $10 \%$ Tukey window. In this way, the resulting $-6 \mathrm{~dB}$ signal bandwidth matches the probe bandwidth $(2.7 \mathrm{MHz})$. The length of the chirp was chosen by considering a tradeoff among SNR gain, depth of the blind zone, and imaging artifacts [45]-[47]. The tone burst, which is also used for B-mode imaging, includes 2.5 cycles of a sinusoid at $3.7 \mathrm{MHz}$. The burst is weighted by a Hamming window to obtain the same $(-6 \mathrm{~dB})$ bandwidth and maximum amplitude of the linear chirp. The Bmode images are obtained by scanning 128 consecutive lines on $64^{\circ}$-wide sectors.

Fig. 3 shows sample one-way fields produced by the system when the tone bursts were transmitted and detected using the HGL008 hydrophone (Onda Corporation, Sunnyvale, CA, USA) moved in a water tank by a home-made 6-axis positioning system. Grating lobes are generally due to the sparsity of active elements, but those visible at $\mathrm{x}=3 \mathrm{~mm}$ on the $x y-p l a n e$ are due to the not connected rows of elements on the y-direction, see Fig. 1.

\section{3) Reception}

In RX, the echo signals received after each TX event are band-pass filtered (cut-off frequencies: 0.25 and $10.0 \mathrm{MHz}$ ), time gain compensated, and analog-to-digital converted at 78 $\mathrm{MHz}$ with 12-bit resolution. Then, they are dynamically beamformed with constant f-number $(\mathrm{F} \#=2)$ by the FPGAs onboard the ULA-OP 256. Finally, the beamformed signals are quadrature demodulated and downsampled by the available DSPs to produce up to 512 output samples. The downsampling factor can be adjusted by the user to cover the range of interest.

\section{4) Matched filtering}

The ULA-OP's DSPs are also in charge of applying matched filtering to the echo-data obtained in Doppler mode. In order to reduce the computational load, matched filtering is performed on baseband data and in the frequency-domain rather than in the time-domain [48]. Baseband operation is not expected to produce any distortion since the chirp duration is limited to 5 $\mu$ s and the active aperture is relatively small [49], [50]. For each $\mathrm{TX} / \mathrm{RX}$ event, pulse compression is thus achieved in 3 steps:
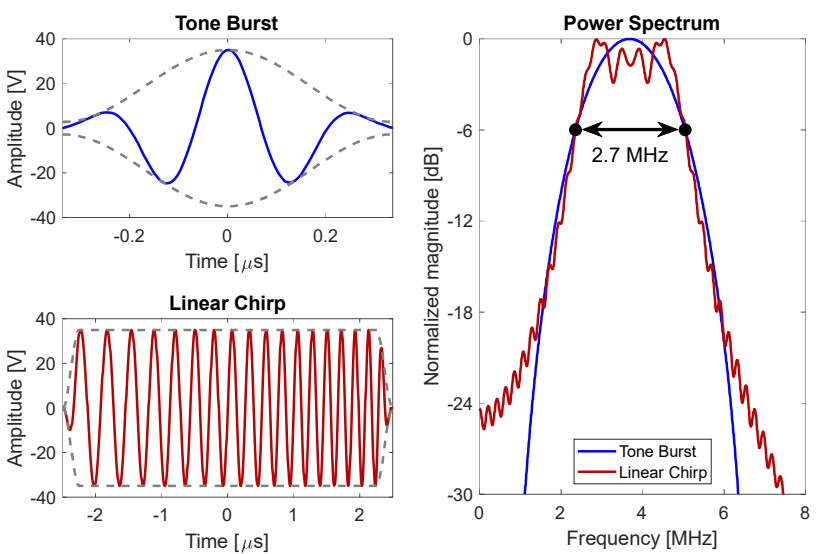

Fig. 2 The excitation signals (left panels) and their power spectra (right panel). On the left panels, the dashed gray lines represent the envelope of the tapering windows. 

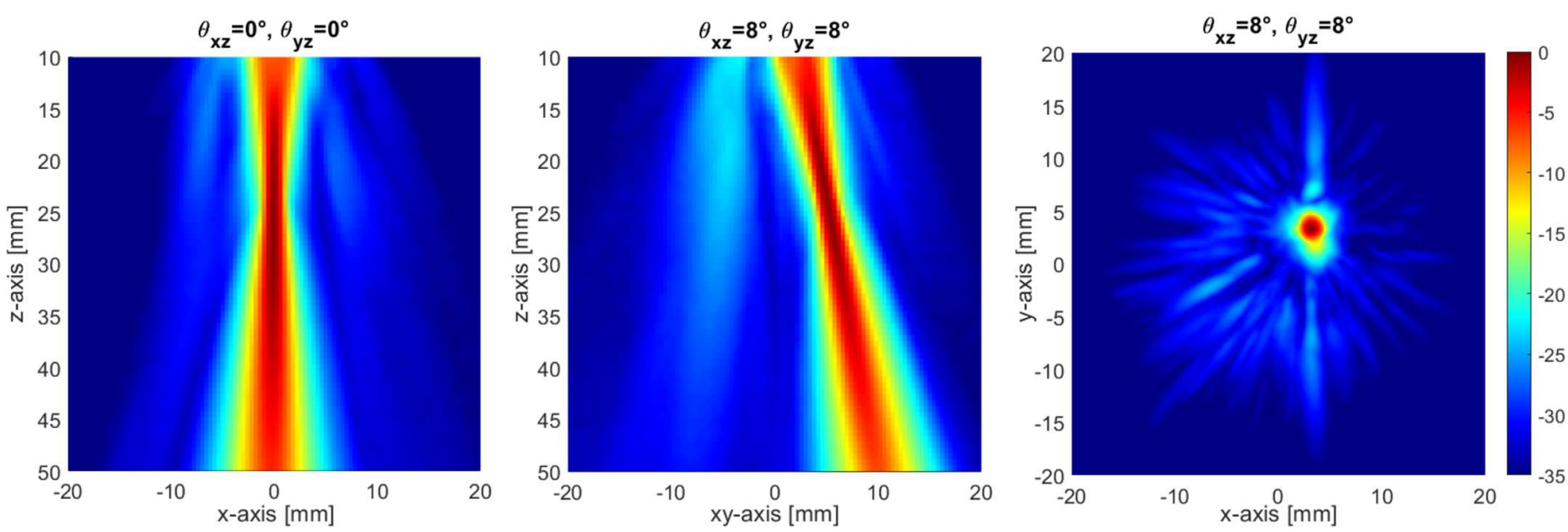

Fig. 3 Examples of experimental one-way fields produced by the 256-element sparse array. In the left panel, the beam was not steered and was acquired on the plane with rotational angle equal to $0^{\circ}$, i.e. the xz-plane. In the middle and right panels, the beam was steered by $8^{\circ}$ on both lateral directions and was acquired on the plane with $45^{\circ}$ rotational angle (center) and on a plane parallel to the probe surface at a depth of $25 \mathrm{~mm}$ (right).

1) Up to 512 (fast-time) beamformed, quadrature demodulated, and downsampled data are transformed, after zero-padding, to the frequency domain through a 1024-point Fast Fourier Transform (FFT).

2) The resulting complex spectra are multiplied by the coefficients of the matched filter. The frequency response of this filter reproduces the complex conjugate spectrum of the TX signal. When needed, the amplitude response can be weighted by a window that partially mismatches the filter while reducing the side-lobes [45], [46], [49].

3) Spectral data are transformed back to the time domain through a 1024-point inverse-FFT and the 512 valid output samples are made available for the next processing stages.

It is worth noting that, for a fair comparison, matched filtering was applied to the data of both the tone burst and the linear chirp.

\section{5) B-mode and Doppler processing}

For B-mode imaging, the moduli of baseband complex data are computed and log-compressed before being transferred to the host PC. The data are finally scan-converted and displayed in grayscale by the $\mathrm{PC}$ real-time interface.

In MSD-mode, the slow-time, baseband, matched filtered samples corresponding to each of 512 different depths along the Doppler line, are FFT-processed by the DSP. The complex moduli of the spectra are then transferred to the host PC. Here they are displayed as color-scale images in which the horizontal axis corresponds to the Doppler frequencies and the vertical axis to the depth of the examined sample volume [7].

The user can also select one of the MSD depths to simultaneously show the corresponding spectrogram as a colorscale image in which the horizontal axis corresponds to time and the vertical axis to the Doppler frequencies.

\section{B. Experimental tests}

Different TX/RX strategies, each dedicated to one of the experimental tests that are described in the following paragraphs, were implemented.

\section{1) Assessment of SNR gain}

In order to prove the effectiveness of real-time pulse compression in 3-D Doppler mode, experiments were conducted on a homemade flow phantom. This consisted of a straight, rigid, cylindrical tube immersed in a water tank and connected to a hydraulic circuit, through which a bloodmimicking fluid was pushed by a programmable peristaltic pump (Watson-Marlow, Falmouth, UK). Specifically:

- The 70-cm long tube, having an inner diameter of $8 \mathrm{~mm}$ and $1-\mathrm{mm}$ thick wall, was made of Rilsan ${ }^{\circledR}$ (Arkema S.A., Colombes, France) - a polyamide and bioplastic member of the nylon family of polymers - characterized by an attenuation coefficient of $2 \mathrm{~dB} / \mathrm{cm} / \mathrm{MHz}$ and sound propagation speed of $2600 \mathrm{~m} / \mathrm{s}$.

- The blood-mimicking fluid consisted of $2.5 \mathrm{~g}$ of $10-\mu \mathrm{m}$ particles of Orgasol $^{\mathbb{B}}$ (Arkema S.A., Colombes, France) - a polyamide powder - suspended in 2 liters of demineralized water with $1 \mathrm{~g}$ of surface-active agent for a uniform distribution of particles [51].

- The fluid flowed at a steady rate of $280 \mathrm{ml} / \mathrm{min}$, from a reservoir into an expansion tank, then through the straight tube, and finally back to the reservoir.

Acquisitions were repeated for 9 different directions of the Doppler line, corresponding to the combinations of the angles $0^{\circ}, 8^{\circ}$, and $16^{\circ}$ in both $\mathrm{xz}$ - and yz-plane.

During these tests, as sketched in Fig. 4, manual setting of the probe position was facilitated by two real-time B-mode images: the first one, reconstructed along the plane with $90^{\circ}$ rotational angle, was used to intercept the cross-section of the tube; the second one, whose rotational angle was programmed to include the Doppler investigation line, was used to ensure a proper alignment between the tube axis and the focal depth of the Doppler line.

To allow comparing the Doppler SNRs achieved by using different TX signals, the ULA-OP 256 was programmed to interleave, at $1 \mathrm{kHz}$ PRF, the following TX events:

1) Sinusoidal burst TX along a scan line of the B-mode image 1 .

2) Linear chirp TX along the Doppler line. 
3) Sinusoidal burst TX along a scan line of the B-mode image 2.

4) Sinusoidal burst $T X$ along the Doppler line.

Slow-time Doppler signals were acquired for an interval of 6 s. In this case, data were post-processed by a Matlab ${ }^{\circledR}$ (The MathWorks, Natick, MA) script that replicates the real-time MSD processing implemented on the ULA-OP 256. The Doppler SNRs obtained with each of the two TX signals were computed as proposed in [47]. Briefly, consecutive MSD frames were averaged so that smooth images, like those in Fig. 5 , were obtained. Here, the region of the Doppler spectrum related to the flow $\left(\mathrm{S}_{\mathrm{ROI}}\right)$, i.e. the region within the $-6 \mathrm{~dB}$ isolines, which includes $n_{S}$ spectra, was semi-automatically segmented. Once the position of the walls was manually pointed, an automatic algorithm fitted the borders of the $-6 \mathrm{~dB}$ isolines with a second-degree polynomial curve. The noise region $\left(\mathrm{N}_{\mathrm{ROI}}\right)$ was selected in the upper portion of the image, covering $n_{N}=50$ depths, where the flow was absent. The SNR was thus estimated as:

$$
S N R=10 \log _{10}\left(\frac{S}{N} \cdot \frac{n_{N}}{n_{S}}\right)
$$

where $S$ and $N$ correspond to the total power measured in $S_{R O I}$ and $N_{R O I}$, respectively.

Finally, for each acquisition, the SNR gain (GSNR) was computed as the difference between the SNRs obtained with the chirp TX and with the sinusoidal burst TX, respectively.

\section{2) MSD investigation along non-isoplanar lines}

In order to prove the capability of assessing blood flow along lines located in different planes, an anthropomorphic phantom [52] was used. Specifically, the phantom mimicked the bifurcation of the carotid artery with stenosis of the internal

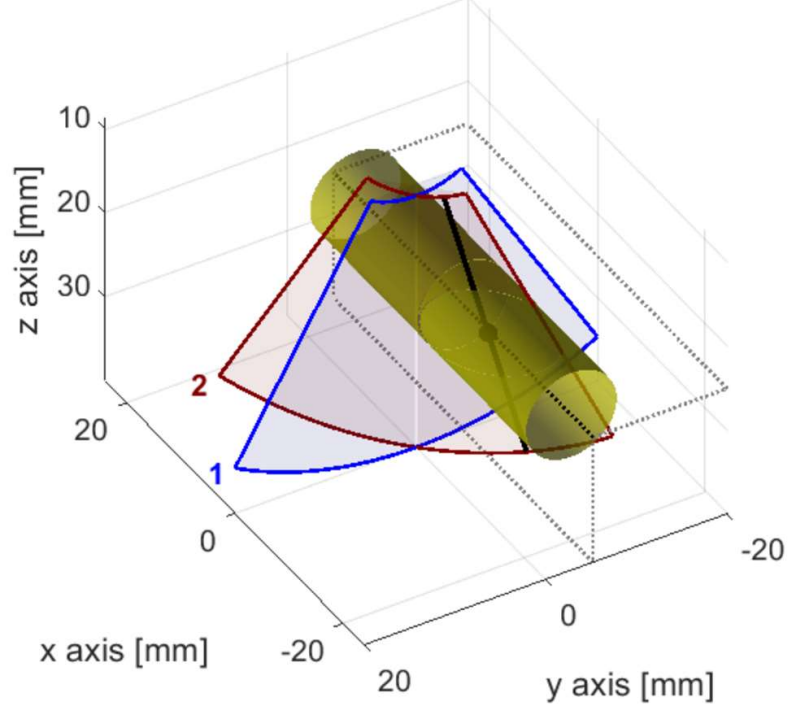

Fig. 4 The reference system and the scanning planes used to facilitate the alignment of the probe with the straight tube. The probe was centered on the origin of the reference system. The first plane, with $90^{\circ}$ rotational angle (blue), was used to intercept the cross section of the tube (yellow). The second plane (red), was used to ensure a proper alignment between the tube axis and the focusing point (black dot) of the (solid black) Doppler line, here steered at $\theta_{\mathrm{xz}}=\theta_{\mathrm{yz}}=16^{\circ}$. Gray dotted lines represent the projection lines of the tube axis along the main planes. carotid artery (ICA). The phantom was inserted in the hydraulic circuit so that the carotid bifurcation lied parallel to the probe surface.

As sketched in Fig. 6, during these tests, two real-time Bmode images, crossing at the level of the common carotid artery (CCA), were shown: the first, coincident with the plane at $25^{\circ}$ rotational angle, reproduced a longitudinal section including the origin of the external carotid artery (ECA); the second one, coincident with the plane with $0^{\circ}$ rotational angle, reproduced a section including the ICA origin.

Two Doppler lines, both focused at $\sim 30 \mathrm{~mm}$ depth, were set on the two imaging planes: line A intercepted the CCA, while line $\mathrm{B}$ was close to the origin of the ICA. The steering angles, on the xz- and yz-plane, were $\left(\theta_{x z}=-14.5^{\circ}, \theta_{y z}=-6.9^{\circ}\right)$ and $\left(\theta_{x z}=-8.6^{\circ}, \theta_{y z}=0.0^{\circ}\right)$ for line $\mathrm{A}$ and $\mathrm{B}$, respectively.

The ULA-OP 256 was here programmed to interleave, at 1 $\mathrm{kHz}$ PRF, the following TX events:

1) Sinusoidal burst TX along a scan line of the B-mode image 1.

2) Linear chirp TX along the Doppler line A.

3) Sinusoidal burst TX along a scan line of the B-mode image 2.

4) Linear chirp TX along the Doppler line B.
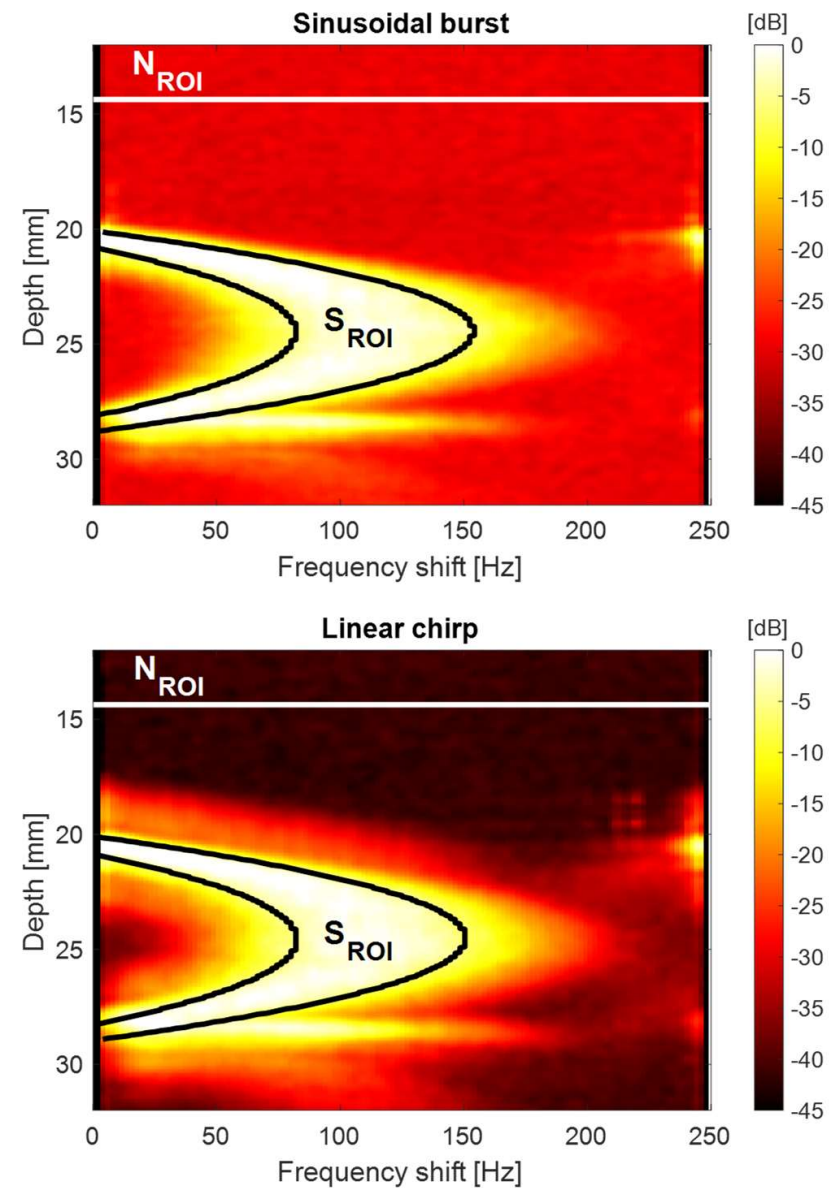

Fig. 5 Examples of averaged MSD images highlighting the regions of the Doppler spectrum related to the flow $\left(\mathrm{S}_{\mathrm{ROI}}\right)$ and to the noise $\left(\mathrm{N}_{\mathrm{ROI}}\right)$. The examples refer to the case with $\theta_{x z}=\theta_{y z}=16^{\circ}$. On top, the MSD image obtained with the sinusoidal burst; on bottom, the one obtained with the linear chirp. 


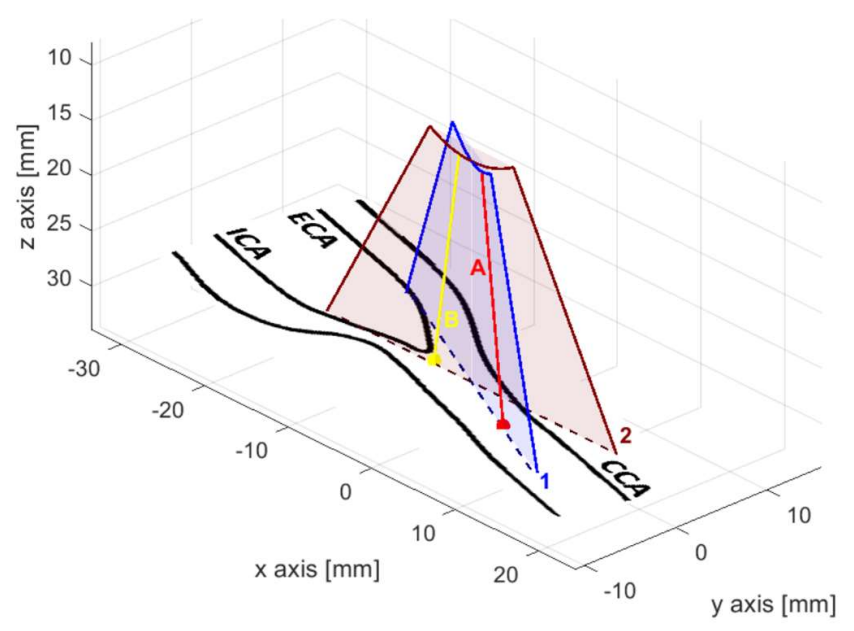

Fig. 6 Schematic view of the carotid bifurcation phantom, placed parallel to the probe surface at roughly $30-\mathrm{mm}$ depth. B-Mode images were obtained along planes 1 and 2. Doppler lines were steered along directions $\mathrm{A}$ and $\mathrm{B}$.

B-mode images and MSD spectra were shown in real-time on the ULA-OP 256 software interface.

\section{3) In vivo experiments}

Two real-time B-mode images, which scan planes at different rotational angles, were made available to facilitate the probe positioning and to allow setting the direction of two Doppler lines during in vivo tests.

The ULA-OP 256 was thus programmed to interleave, at 3 kHz PRF, 6 TX events:

1) Sinusoidal burst $\mathrm{TX}$ along a scan line of the B-mode image 1.

2) Linear chirp TX along the Doppler line A.

3) Linear chirp TX along the Doppler line B.

4) Sinusoidal burst TX along a scan line of the B-mode image 2 .

5) Linear chirp TX along the Doppler line A.

6) Linear chirp TX along the Doppler line B.

B-mode images, MSD profiles, and spectrograms from a selected depth were shown in real-time on the ULA-OP 256 software interface.

In this interleaved mode, the mechanical index (MI), the thermal index for soft tissues at the surface (TIS ${ }_{\mathrm{as}}$ ), and the temperature rise at the probe surface $(\Delta \mathrm{T})$ were estimated. The MI was 0.12 and 0.10 for chirp and sinusoidal burst, respectively. The $\mathrm{TIS}_{\mathrm{as}}$ was always lower than 0.01 , while $\Delta \mathrm{T}$ was $4^{\circ} \mathrm{C}$, after a 30 -minute continuous operation in still air. Then, preliminary acquisitions were performed on the carotid arteries of healthy volunteers.

\section{RESULTS}

\section{1) Assessment of SNR gain}

Fig. 5 shows two MSD images obtained by transmitting the sinusoidal burst and the linear chirp, respectively. The Doppler line was set at $\theta_{x z}=\theta_{y z}=16^{\circ}$. Qualitatively, the images do not show a significant difference in the spectrum shape. Also, both spectra show the same artifact due to the saturation of beamformed signals from the second wall of the plastic tube. However, an improved SNR is clearly achieved with pulse compression. Indeed, since both images were normalized to the respective overall maximum intensity, the noise level obtained with the chirp appears considerably lower than that of the sinusoidal burst. Equivalent results were obtained for all tested steering conditions and the estimated SNRs and GSNRs are summarized in TABLE I. On average (see the rightmost column), the estimated SNRs were $19.6 \mathrm{~dB}$ for the sinusoidal burst and $31.0 \mathrm{~dB}$ for the linear chirp, which corresponded to an average GSNR of $11.4 \mathrm{~dB}$.

\section{2) MSD investigation along non-isoplanar lines}

Fig. 7 reports a screenshot of the ULA-OP 256 interface obtained during experiments conducted on the carotid artery bifurcation phantom. The sector sketched in Fig. 6 is here shown as a B-mode image in Panel 1. It reproduces a region from the origin of the ECA (on the left) to the bulb in the CCA (on the right). Correspondingly, a slight increase in the vessel diameter can be observed. The (red) Doppler line here intercepted the beginning of the CCA bulb, producing MSD profiles like the one in panel A, showing a parabolic-like shape in the direction of positive Doppler shifts.

The B-mode sector shown in Panel 2 reproduces the phantom from the origin of the ICA (on the center-left) to the external wall of the CCA (on the center-right). The (yellow) Doppler line was steered on the origin of the ICA just before the stenosis. The MSD profile (panel B) is parabolic-like also here but, compared to the one in panel A, it looks "full" since the Doppler angle was close to $90^{\circ}$ [53] and the spectral broadening was thus high. It is worth highlighting that, in this interleaved setting, a $\mathrm{PRF}=1 \mathrm{kHz}$ corresponded to a Doppler sampling rate of 250 $\mathrm{Hz}$, which was purposely adapted to get as close as possible to the aliasing limit on panel $\mathrm{A}$ and, hence, to exploit the whole available Doppler band.

\section{3) In vivo experiments}

Fig. 8 shows two examples of the ULA-OP 256 interface frozen during real-time operation on healthy volunteers, which were extracted from the supplementary accompanying video clips. In these examples, the bi-planar B-mode volume rate, limited by the PRF $(3 \mathrm{kHz})$, the number of interleaved transmission events (6), and the number of lines per frame (128), was $3.9 \mathrm{~Hz}$, which is quite low but sufficient to support

TABLE I ESTIMATED SNR AND GSNR

\begin{tabular}{|c|c|c|c|c|c|c|c|c|c|c|c|}
\hline & \multirow{2}{*}{$\begin{array}{l}\boldsymbol{\theta}_{x z} \\
\boldsymbol{\theta}_{y z}\end{array}$} & \multicolumn{3}{|c|}{$\mathbf{0}^{\circ}$} & \multicolumn{3}{|c|}{$8^{\circ}$} & \multicolumn{3}{|c|}{$16^{\circ}$} & \multirow{2}{*}{$\begin{array}{c}\text { Avg } \pm \text { Std } \\
\text { values }\end{array}$} \\
\hline & & $\mathbf{0}^{\circ}$ & $8^{\circ}$ & $16^{\circ}$ & $0^{\circ}$ & $8^{\circ}$ & $16^{\circ}$ & $0^{\circ}$ & $8^{\circ}$ & $16^{\circ}$ & \\
\hline \multirow{2}{*}{ SNR [dB] } & "Sinusoidal burst & 19.5 & 20.0 & 18.8 & 21.9 & 20.9 & 19.2 & 19.9 & 18.8 & 17.2 & $19.6 \pm 1.3$ \\
\hline & Linear chirp & 30.4 & 31.6 & 29.9 & 33.2 & 32.3 & 30.4 & 31.1 & 31.6 & 28.6 & $31.0 \pm 1.4$ \\
\hline \multicolumn{2}{|c|}{ GSNR [dB] } & 10.9 & 11.6 & 11.1 & 11.3 & 11.4 & 11.2 & 11.2 & 12.8 & 11.4 & $11.4 \pm 0.6$ \\
\hline
\end{tabular}


probe positioning. On the other hand, the update rate of MSD profiles was arbitrarily set to $40 \mathrm{~Hz}$, which is high enough for real-time display, but, considering the computational power of the DSP, it could have been increased up to hundreds of $\mathrm{Hz}$.

In the first example 国, Fig. 8 left, the delta between the rotational angles of the two B-mode images was set to $90^{\circ}$. In this way, the longitudinal and the cross-section of the CCA were scanned. The Doppler line A was steered by $10^{\circ}$ within plane A, while line B was set perpendicular to the probe surface.

Both MSD profiles have an M-like shape, which is typical during late systole [54]. In panel B, in particular, a strong clutter from the walls is visible and the spectra look "full", down to 0 $\mathrm{Hz}$, as usual when the Doppler angle is close to $90^{\circ}$. Vice versa, the profiles along the Doppler line A show a wider spectral window, as expected at a lower Doppler angle.

In the second example rotational angles of the two B-mode images was set to $60^{\circ}$. To simulate a non-optimal positioning of the probe, it was slightly shifted with respect to the axis of the CCA, but both Doppler lines were roughly focused on the CCA axis. The two B-mode images show two cross-sections of the CCA, which take the form of ellipses. Both MSD profiles have a parabolic-like shape, as expected during diastole, and show a strong wall clutter. Moreover, they have opposite Doppler shifts: negative along line $\mathrm{A}$ and positive along line $\mathrm{B}$, as expected from the schematic view sketched in Fig. 8 center-bottom. Finally, also in this case, both the MSD profiles and the sonograms are "full", due to the almost $90^{\circ}$ Doppler angles.

\section{Discussion AND CONCLUSION}

In this article, we have presented the first system capable of performing real-time spectral Doppler from any of the points in the volume, scanned by a 2-D spiral array. The system was
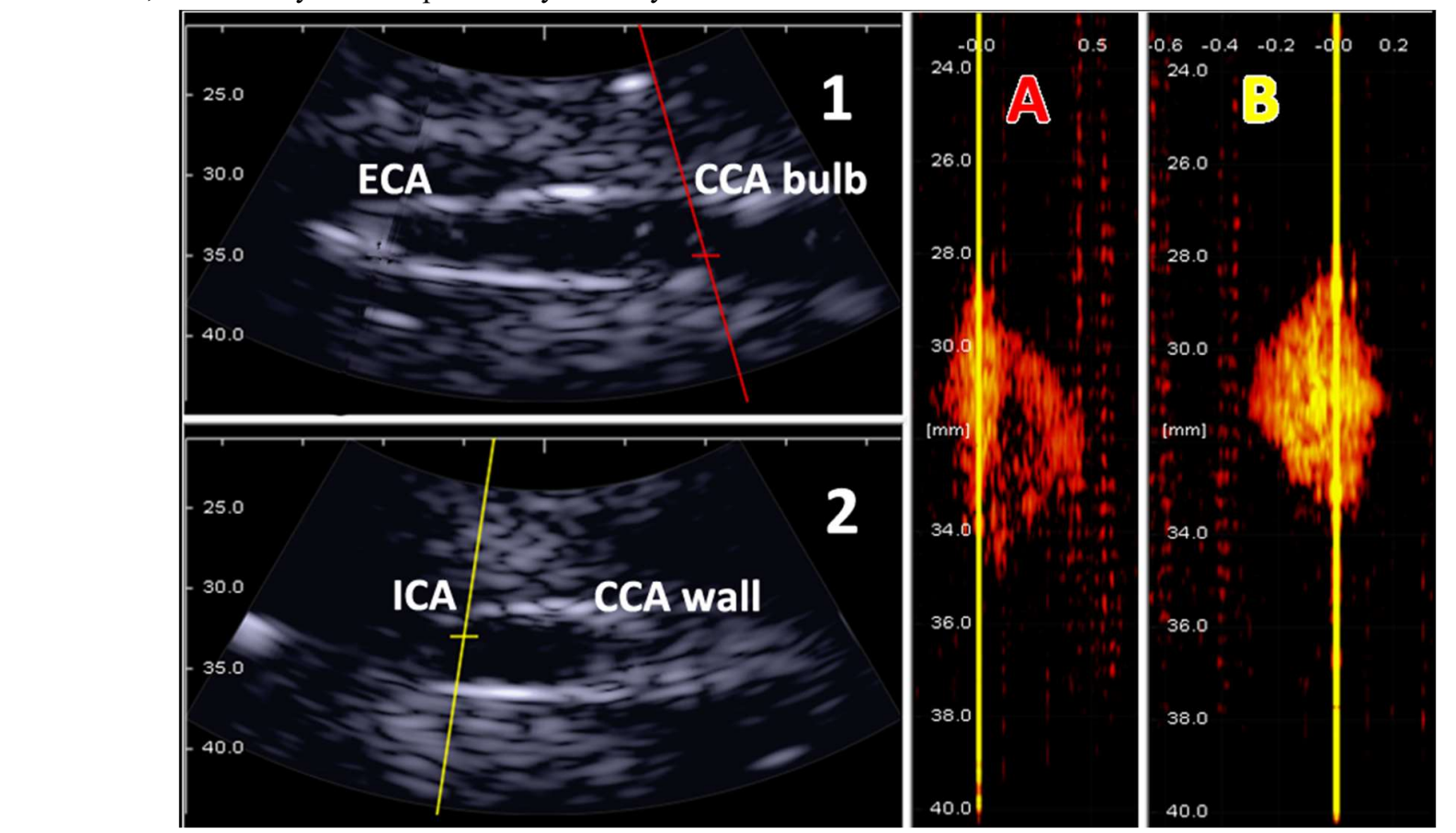

Fig. 7 Screenshot of the ULA-OP 256 interface during real-time operation. B-Mode images $(1,2)$ and MSD profiles (A, B) were obtained as described in the

schematic view of the carotid bifurcation phantom in Fig. 6 . The dynamic range of B-mode images was $30 \mathrm{~dB}$, while that of MSD profiles was $24 \mathrm{~dB}$ based on the ULA-OP 256 research scanner and on a prototype spiral probe, designed by selecting 256 elements from a 1024element gridded array. The MSD modality, which represents an extension of classic PW Doppler to multiple aligned sample volumes, has been implemented. We also tested the effectiveness of coded transmission and pulse compression in reception to compensate for the low achievable SNR due to the limited number of elements.

The performance of the system was quantitatively assessed on experimental data acquired from flow phantoms based either on a straight tube and on an anthropomorphic phantom of the CCA. Finally, for a qualitative assessment of the system performance in-vivo, real-time images were collected on healthy volunteers.

Different interleaving sequences, depending on the application of interest, were implemented. For example, the TX sequence for B-mode imaging was interleaved with the transmission of: (1) different Doppler pulses (i.e. a sinusoidal burst and a linear chirp) along the same investigation line, for SNR assessment; (2) chirp Doppler pulses along investigation lines within different scan planes, for a qualitative blood flow assessment in the anthropomorphic phantom and in-vivo. Together with the two B-mode images, used to facilitate probe positioning, two MSD profiles with (Fig. 8), or without (Fig. 7) the spectrograms from selected depths were simultaneously displayed. This capability could be used to observe the flow dynamics along non-isoplanar Doppler lines, e.g. to simultaneously assess the flow before and after a stenosis, an examination of tortuous arteries: the bi-plane scan could ease the optimal probe positioning and the rapid, real-time, setting of the direction of the Doppler lines of interest. aneurysm, or an aortic stent. Also, it could facilitate the 
In [40] it was experimentally demonstrated, through off-line calculations, that the use of sparse, rather than full arrays, neither affects the shape nor the mean frequency of the Doppler spectrum, but it impairs the SNR. The results presented in this work have shown the effectiveness of pulse compression in SNR enhancement. Specifically, the use of a linear chirp [47], instead of a sinusoidal burst, has brought to an SNR increase of $11.4 \mathrm{~dB}$ (see TABLE I), which was quite stable (standard deviation equal to $0.6 \mathrm{~dB}$ ) for all tested steering angles. Also, in the tested conditions $(\mathrm{B}=2.7 \mathrm{MHz}, \mathrm{T}=5 \mu \mathrm{s})$, the average SNR increase resulted in $11.3 \mathrm{~dB}$, very close to the theoretical value $\left(10 \log _{10}(B \cdot T)\right)$ [49]. Such gain partially compensated the limited SNR associated with the use of a reduced number of elements (here 256) typical of sparse arrays. Considering that any possible doubling of such number is expected to increase the SNR by $9 \mathrm{~dB}$ (6 in TX and 3 in RX) [40], the pulse compression gain here allowed obtaining a performance better than that achievable with 512 elements excited by standard pulses. In pulsed wave Doppler applications, the SNR could also be increased by exploiting long, narrowband pulses, with conflicting implications on transit time and velocity gradient broadening, respectively. Nevertheless, lengthening the pulses is not advisable in applications where the range resolution is important, e.g. in multi-gate spectral Doppler and color flow imaging. Pulse compression thus represents a good means to achieve high SNR while using wideband, i.e. high resolution, signals.

Even if, in general, the transmission of long linear chirp signals for extended exposure times might rise thermal effects [47], on both probe and skin surfaces, this did not happen in our tests. The relatively low excitation voltage $( \pm 35 \mathrm{~V})$ and the sparse distribution of the active elements most likely facilitated heat dissipation. Therefore, the excitation voltage could be further increased, but in our case, we preferred to stay below the maximum voltage limit $( \pm 45 \mathrm{~V})$ suggested by the probe manufacturer.

In this paper, a sparse spiral array was associated with a 256channel research system to detect, in real-time, the flow profiles along non-isoplanar investigation lines in phantom (Fig. 7) and in vivo (Fig. 8). The profiles, as expected, show different shapes, depending on the Doppler angle and investigated region. In vivo, in particular, although tissue attenuation further impaired the SNR, the sonograms and the profiles exhibited the expected spectral windows and the same physiological trend that can be observed with linear arrays [54].

All benefits and limitations of 2-D spectral Doppler can be extended to 3-D. For example, quantitative information on blood flow velocity could be, to some extent, obtained through simple angle correction or through vector flow imaging techniques that exploit either the Doppler bandwidth [55], [56], multiple transmission/reception beams [16], [57], [58], or speckle tracking [20], [23].

The results also highlight some limitations of the current system. The MSD images in panels A, B of Fig. 7, present secondary profiles whose depth and frequency are slightly shifted with respect to the main Doppler profile. These profiles can be attributed to the characteristics of the transmitted fields, which, in addition to the main steered beam, also show relatively high (up to -20dB) grating lobes, as visible in Fig. 3. Furthermore:

1) The probe characteristics were not ideal for the investigation of superficial vessels: the central frequency was too low and the aperture size too small $(<1 \mathrm{~cm})$ to achieve good spatial resolution.

2) The relatively wide elements and the big average pitch limit the probe steering capability, in terms of reduced sensitivity and increased grating lobe level, respectively. This, in turn, limits the effective size of the region of
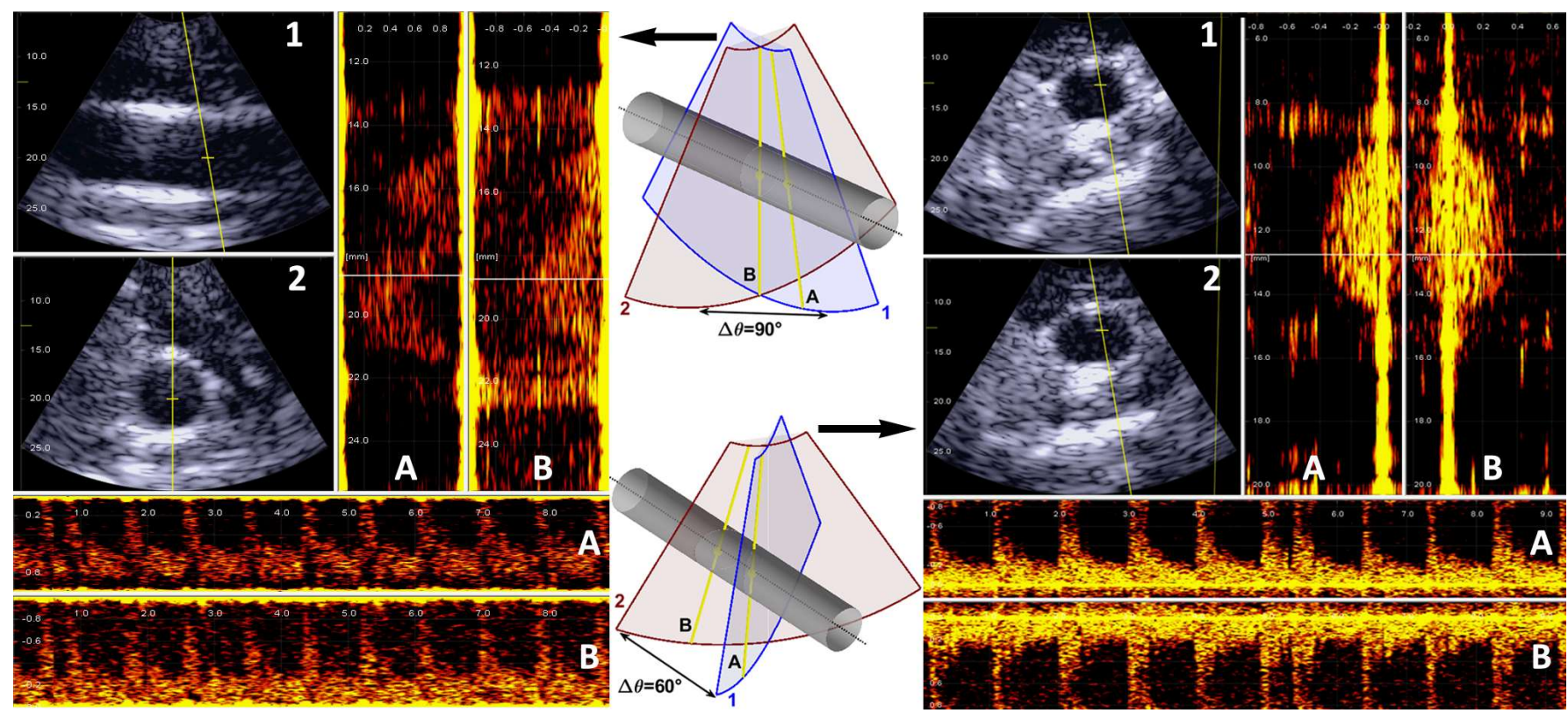

Fig. 8 Two examples of the ULA-OP 256 interface during real-time in-vivo operation on the common carotid artery of healthy volunteers (left and right). B-Mode images $(1,2)$, MSD profiles and related sonograms $(\mathrm{A}, \mathrm{B})$ were obtained as described in the schematic views (center), where the common carotid artery is sketched as a gray cylinder. The dynamic range of B-mode images was $30 \mathrm{~dB}$, while that of MSD profiles was $24 \mathrm{~dB}$. 
interest.

3) The navigation system based on two B-mode images, although capable of working at reasonably high frame rates, does not facilitate the detection of the vessels of interest, as a full 3-D rendering could do.

For clinical examinations of shallow vessels, wider apertures, linear scans, and higher frequencies would be preferable. The presented configuration might be suitable for the investigation of the carotid artery or the jugular vein as well as for echocardiography [43] and abdominal vascular applications. Nevertheless, to make the system suitable for clinical tests, the following features should be included:

1) Integration of in-probe pre-amplifiers to further improve the SNR and penetration depth [59].

2) A better navigation system, which could include a Cmode display and, possibly, an automatic system to determine the rotational angles of the imaging planes, adapting them to the specific anatomy of the subject (e.g. based on machine learning [60]).

In conclusion, considering the above aspects, the results of this paper are really encouraging and confirm that sparse arrays are a feasible option for the implementation of 3-D imaging/Doppler systems. At a relatively cheap cost, they may allow physicians to simultaneously assess the dynamics of blood flow within an entire volume of interest, at a high temporal resolution, and on multiple non-isoplanar directions simultaneously. Moreover, the 3-D morphological view will provide more degrees of freedom and less room for errors in probe positioning.

\section{REFERENCES}

[1] Ph. D. Evans D. H. and W. N. McDicken, Doppler ultrasound: physics, instrumentation, and signal processing, 2nd ed. Chichester; New York: J. Wiley, 2000.

[2] E. G. Grant et al., "Carotid Artery Stenosis: Gray-Scale and Doppler US Diagnosis-Society of Radiologists in Ultrasound Consensus Conference,' Radiology, vol. 229, no. 2, pp. 340-346, Nov. 2003, doi: 10.1148/radiol.2292030516.

[3] D. Maulik, Doppler Ultrasound in Obstetrics and Gynecology, 2nd rev. and enlarged edition. Berlin Heidelberg New York: Springer, 2005.

[4] I. M. Coman and B. A. Popescu, "Shigeo Satomura: 60 years of Doppler ultrasound in medicine," Cardiovasc. Ultrasound, vol. 13, Dec. 2015, doi: 10.1186/s12947-015-0042-3.

[5] Z. Nuffer, A. Rupasov, N. Bekal, J. Murtha, and S. Bhatt, "Spectral Doppler ultrasound of peripheral arteries: a pictorial review," Clin. Imaging, vol. 46, pp. 91-97, Nov. 2017, doi: 10.1016/j.clinimag.2017.07.007.

[6] J. A. Jensen et al., "History and Latest Advances in Flow Estimation Technology: From 1-D in 2-D to 3-D in 4-D," in 2019 IEEE International Ultrasonics Symposium (IUS), Oct. 2019, pp. 1041-1050, doi: 10.1109/ULTSYM.2019.8926210.

[7] P. Tortoli, G. Bambi, F. Guidi, and R. Muchada, "Toward a better quantitative measurement of aortic flow," Ultrasound Med. Biol., vol. 28, no. 2, pp. 249-257, Feb. 2002, doi: 10.1016/S0301-5629(01)00462-8.

[8] J. Provost, C. Papadacci, C. Demene, J. L. Gennisson, M. Tanter, and M. Pernot, "3-D ultrafast doppler imaging applied to the noninvasive mapping of blood vessels in Vivo," IEEE Trans. Ultrason. Ferroelectr. Freq. Control, vol. 62, no. 8, pp. 1467-1472, Aug. 2015, doi: 10.1109/TUFFC.2015.007032.

[9] H. Bouzari et al., "Curvilinear 3-D Imaging Using Row-ColumnAddressed 2-D Arrays With a Diverging Lens: Feasibility Study," IEEE Trans. Ultrason. Ferroelectr. Freq. Control, vol. 64, no. 6, pp. 978-988, Jun. 2017, doi: 10.1109/TUFFC.2017.2687521.

[10] J. Zhou et al., "High-Volume-Rate 3-D Ultrasound Imaging Based on Synthetic Aperture Sequential Beamforming With Chirp-Coded Excitation," IEEE Trans. Ultrason. Ferroelectr. Freq. Control, vol. 65, no. 8, pp. 13461358, Aug. 2018, doi: 10.1109/TUFFC.2018.2839085.
[11] S. Fiorentini, L. M. Saxhaug, T. G. Bjåstad, E. Holte, H. Torp, and J. Avdal, "Maximum Velocity Estimation in Coronary Arteries Using 3-D Tracking Doppler," IEEE Trans. Ultrason. Ferroelectr. Freq. Control, vol. 65, no. 7, pp. 1102-1110, Jul. 2018, doi: 10.1109/TUFFC.2018.2827241.

[12] J. Udesen, F. Gran, K. L. Hansen, J. A. Jensen, C. Thomsen, and M. B. Nielsen, "High frame-rate blood vector velocity imaging using plane waves: simulations and preliminary experiments," IEEE Trans. Ultrason. Ferroelectr. Freq. Control, vol. 55, no. 8, pp. 1729-1743, Aug. 2008, doi: 10.1109/TUFFC.2008.858.

[13] M. Lenge, A. Ramalli, E. Boni, H. Liebgott, C. Cachard, and P. Tortoli, "High-frame-rate 2-D vector blood flow imaging in the frequency domain," IEEE Trans. Ultrason. Ferroelectr. Freq. Control, vol. 61, no. 9, pp. 15041514, Sep. 2014, doi: 10.1109/TUFFC.2014.3064.

[14] J. A. Jensen, S. I. Nikolov, A. C. H. Yu, and D. Garcia, "Ultrasound Vector Flow Imaging-Part I: Sequential Systems," IEEE Trans. Ultrason. Ferroelectr. Freq. Control, vol. 63, no. 11, pp. 1704-1721, Nov. 2016, doi: 10.1109/TUFFC.2016.2600763.

[15] J. Avdal, L. Løvstakken, H. Torp, and I. K. Ekroll, "Combined 2-D Vector Velocity Imaging and Tracking Doppler for Improved Vascular Blood Velocity Quantification," IEEE Trans. Ultrason. Ferroelectr. Freq. Control, vol. 64, no. 12, pp. 1795-1804, Dec. 2017, doi: 10.1109/TUFFC.2017.2757600.

[16] S. Ricci, A. Ramalli, L. Bassi, E. Boni, and P. Tortoli, "Real-Time Blood Velocity Vector Measurement Over a 2-D Region," IEEE Trans. Ultrason. Ferroelectr. Freq. Control, vol. 65, no. 2, pp. 201-209, Feb. 2018, doi: 10.1109/TUFFC.2017.2781715.

[17] P. Tortoli, M. Lenge, D. Righi, G. Ciuti, H. Liebgott, and S. Ricci, "Comparison of carotid artery blood velocity measurements by vector and standard Doppler approaches," Ultrasound Med. Biol., vol. 41, no. 5, pp. 1354 1362, May 2015, doi: 10.1016/j.ultrasmedbio.2015.01.008.

[18] J. A. Jensen, S. I. Nikolov, A. C. H. Yu, and D. Garcia, "Ultrasound Vector Flow Imaging - Part I: Sequential Systems," IEEE Trans. Ultrason. Ferroelectr. Freq. Control, vol. 63, no. 11, pp. 1704-1721, Nov. 2016, doi: 10.1109/TUFFC.2016.2600763.

[19] M. Correia, J. Provost, M. Tanter, and M. Pernot, "4D ultrafast ultrasound flow imaging: in vivo quantification of arterial volumetric flow rate in a single heartbeat," Phys. Med. Biol., vol. 61, no. 23, p. L48, 2016, doi: 10.1088/00319155/61/23/L48.

[20] S. Holbek, T. L. Christiansen, M. B. Stuart, C. Beers, E. V. Thomsen, and J. A. Jensen, "3-D Vector Flow Estimation With Row-Column-Addressed Arrays," IEEE Trans. Ultrason. Ferroelectr. Freq. Control, vol. 63, no. 11, pp. 1799-1814, Nov. 2016, doi: 10.1109/TUFFC.2016.2582536.

[21] S. Holbek et al., "Ultrasonic 3-D Vector Flow Method for QuantitativeIn VivoPeak Velocity and Flow Rate Estimation," IEEE Trans. Ultrason. Ferroelectr. Freq. Control, vol. 64, no. 3, pp. 544-554, Mar. 2017, doi: 10.1109/TUFFC.2016.2639318.

[22] M. S. Wigen et al., "4-D Intracardiac Ultrasound Vector Flow ImagingFeasibility and Comparison to Phase-Contrast MRI," IEEE Trans. Med. Imaging, vol. 37, no. 12, pp. 2619-2629, Dec. 2018, doi: 10.1109/TMI.2018.2844552.

[23] S. Rossi, A. Ramalli, F. Fool, and P. Tortoli, "High-Frame-Rate 3-D Vector Flow Imaging in the Frequency Domain," Appl. Sci., vol. 10, no. 15, Art. no. 15, Jan. 2020, doi: 10.3390/app10155365.

[24] A. Thrush and T. Hartshorne, Peripheral Vascular Ultrasound: How, Why and When. Churchill Livingstone, 1999.

[25] B. Savord and R. Solomon, "Fully sampled matrix transducer for real time 3D ultrasonic imaging," in 2003 IEEE Ultrasonics Symposium (IUS), 2003, vol. 1, pp. 945-953, doi: 10.1109/ULTSYM.2003.1293556.

[26] G. Matrone, A. S. Savoia, M. Terenzi, G. Caliano, F. Quaglia, and G. Magenes, "A volumetric CMUT-based ultrasound imaging system simulator with integrated reception and $\mu$-beamforming electronics models," IEEE Trans. Ultrason. Ferroelectr. Freq. Control, vol. 61, no. 5, pp. 792-804, May 2014, doi: 10.1109/TUFFC.2014.6805693.

[27] "xMATRIX Ultrasound Technology | Philips Ultrasound." https://www.usa.philips.com/healthcare/resources/feature-detail/xmatrix (accessed Aug. 30, 2020).

[28] M. Mozaffarzadeh et al., "Receive/Transmit Aperture Selection for 3D Ultrasound Imaging with a 2D Matrix Transducer," Appl. Sci., vol. 10, no. 15, Art. no. 15, Jan. 2020, doi: 10.3390/app10155300.

[29] R. E. Davidsen, J. A. Jensen, and S. W. Smith, "Two-dimensional random arrays for real time volumetric imaging," Ultrason. Imaging, vol. 16, no. 3, pp. 143-163, Jul. 1994.

[30] A. Austeng and S. Holm, "Sparse 2-D arrays for 3-D phased array imaging - design methods," IEEE Trans. Ultrason. Ferroelectr. Freq. Control, vol. 49, no. 8, pp. 1073-1086, Aug. 2002, doi: 10.1109/TUFFC.2002.1026019. 
[31] O. T. von Ramm, S. W. Smith, and Jr. Pavy, H.G., "High-speed ultrasound volumetric imaging system. II. Parallel processing and image display," IEEE Trans. Ultrason. Ferroelectr. Freq. Control, vol. 38, no. 2, pp. 109-115, Mar. 1991, doi: 10.1109/58.68467.

[32] O. Martínez-Graullera, C. J. Martín, G. Godoy, and L. G. Ullate, "2D array design based on Fermat spiral for ultrasound imaging," Ultrasonics, vol. 50, no. 2, pp. 280-289, Feb. 2010, doi: 10.1016/j.ultras.2009.09.010.

[33] R. L. Haupt, "Thinned arrays using genetic algorithms," IEEE Trans. Antennas Propag., vol. 42, no. 7, pp. 993-999, Jul. 1994, doi: $10.1109 / 8.299602$

[34] A. Trucco, "Thinning and weighting of large planar arrays by simulated annealing," IEEE Trans. Ultrason. Ferroelectr. Freq. Control, vol. 46, no. 2 , pp. 347-355, Mar. 1999, doi: 10.1109/58.753023.

[35] P. Chen, Y. Zheng, and W. Zhu, "Optimized Simulated Annealing Algorithm for Thinning and Weighting Large Planar Arrays in Both Far-Field and Near-Field," IEEE J. Ocean. Eng., vol. 36, no. 4, pp. 658-664, Oct. 2011 , doi: 10.1109/JOE.2011.2164957.

[36] B. Diarra, M. Robini, P. Tortoli, C. Cachard, and H. Liebgott, "Design of Optimal 2-D Nongrid Sparse Arrays for Medical Ultrasound," IEEE Trans. Biomed. Eng., vol. 60, no. 11, pp. 3093-3102, Nov. 2013, doi 10.1109/TBME.2013.2267742.

[37] E. Roux, A. Ramalli, P. Tortoli, C. Cachard, M. C. Robini, and H. Liebgott, "2-D Ultrasound Sparse Arrays Multidepth Radiation Optimization Using Simulated Annealing and Spiral-Array Inspired Energy Functions," IEEE Trans. Ultrason. Ferroelectr. Freq. Control, vol. 63, no. 12, pp. 2138 2149, Dec. 2016, doi: 10.1109/TUFFC.2016.2602242.

[38] E. Roux, A. Ramalli, H. Liebgott, C. Cachard, M. C. Robini, and P. Tortoli, "Wideband 2-D Array Design Optimization With Fabrication Constraints for 3-D US Imaging," IEEE Trans. Ultrason. Ferroelectr. Freq. Control, vol. 64, no. 1, pp. 108-125, Jan. 2017, doi: 10.1109/TUFFC.2016.2614776.

[39] A. Ramalli, E. Boni, A. S. Savoia, and P. Tortoli, "Density-tapered spiral arrays for ultrasound 3-D imaging," IEEE Trans. Ultrason. Ferroelectr. Freq. Control, vol. 62, no. 8, pp. 1580-1588, Aug. 2015, doi: 10.1109/TUFFC.2015.007035.

[40] P. Mattesini, A. Ramalli, L. Petrusca, O. Basset, H. Liebgott, and P. Tortoli, "Spectral Doppler Measurements With 2-D Sparse Arrays," IEEE Trans. Ultrason. Ferroelectr. Freq. Control, vol. 67, no. 2, pp. 278-285, Feb. 2020, doi: 10.1109/TUFFC.2019.2944090.

[41] E. Boni et al., "Architecture of an Ultrasound System for Continuous Real-Time High Frame Rate Imaging," IEEE Trans. Ultrason. Ferroelectr. Freq. Control, vol. 64, no. 9, pp. 1276-1284, Sep. 2017, doi: 10.1109/TUFFC.2017.2727980.

[42] E. Boni, A. C. H. Yu, S. Freear, J. A. Jensen, and P. Tortoli, "Ultrasound Open Platforms for Next-Generation Imaging Technique Development," IEEE Trans. Ultrason. Ferroelectr. Freq. Control, vol. 65, no. 7, pp. 1078-1092, Jul. 2018, doi: 10.1109/TUFFC.2018.2844560.

[43] A. Ramalli et al., "High-Frame-Rate Tri-Plane Echocardiography With Spiral Arrays: From Simulation to Real-Time Implementation," IEEE Trans. Ultrason. Ferroelectr. Freq. Control, vol. 67, no. 1, pp. 57-69, Jan. 2020, doi: 10.1109/TUFFC.2019.2940289.

[44] S. Harput et al., "3-D Super-Resolution Ultrasound Imaging With a 2-D Sparse Array," IEEE Trans. Ultrason. Ferroelectr. Freq. Control, vol. 67, no. 2, pp. 269-277, Feb. 2020, doi: 10.1109/TUFFC.2019.2943646.

[45] R. Y. Chiao and Xiaohui Hao, "Coded excitation for diagnostic ultrasound: a system developer's perspective," IEEE Trans. Ultrason. Ferroelectr. Freq. Control, vol. 52, no. 2, pp. 160-170, Feb. 2005, doi: 10.1109/TUFFC.2005.1406543.
[46] A. Ramalli, F. Guidi, E. Boni, and P. Tortoli, "A real-time chirp-coded imaging system with tissue attenuation compensation," Ultrasonics, vol. 60, pp. 65-75, Jul. 2015, doi: 10.1016/j.ultras.2015.02.013.

[47] A. Ramalli, E. Boni, A. Dallai, F. Guidi, S. Ricci, and P. Tortoli, "Coded Spectral Doppler Imaging: From Simulation to Real-Time Processing," IEEE Trans. Ultrason. Ferroelectr. Freq. Control, vol. 63, no. 11, pp. 1815-1824, Nov. 2016, doi: 10.1109/TUFFC.2016.2573720.

[48] C. Yoon, W. Lee, J. Chang, T. Song, and Y. Yoo, "An efficient pulse compression method of chirp-coded excitation in medical ultrasound imaging," IEEE Trans. Ultrason. Ferroelectr. Freq. Control, vol. 60, no. 10, pp. $2225-$ 2229, 2013, doi: 10.1109/TUFFC.2013.2815.

[49] T. Misaridis and J. A. Jensen, "Use of modulated excitation signals in medical ultrasound. Part I: basic concepts and expected benefits," IEEE Trans. Ultrason. Ferroelectr. Freq. Control, vol. 52, no. 2, pp. 177-191, Feb. 2005, doi: 10.1109/TUFFC.2005.1406545.

[50] T. Misaridis and J. A. Jensen, "Use of modulated excitation signals in medical ultrasound. Part II: design and performance for medical imaging applications," IEEE Trans. Ultrason. Ferroelectr. Freq. Control, vol. 52, no. 2, pp. 192-207, Feb. 2005, doi: 10.1109/TUFFC.2005.1406546.

[51] K. V. Ramnarine, D. K. Nassiri, P. R. Hoskins, and J. Lubbers, "Validation of a New Blood-Mimicking Fluid for Use in Doppler Flow Test Objects," Ultrasound Med. Biol., vol. 24, no. 3, pp. 451-459, Mar. 1998, doi: 10.1016/S0301-5629(97)00277-9.

[52] S. S. M. Lai, B. Y. S. Yiu, A. K. K. Poon, and A. C. H. Yu, "Design of Anthropomorphic Flow Phantoms Based on Rapid Prototyping of Compliant Vessel Geometries," Ultrasound Med. Biol., vol. 39, no. 9, pp. 1654-1664, Sep. 2013, doi: 10.1016/j.ultrasmedbio.2013.03.015

[53] P. Tortoli, G. Guidi, and P. Pignoli, "Transverse doppler spectral analysis for a correct interpretation of flow sonograms," Ultrasound Med. Biol., vol. 19, no. 2, pp. 115-121, Jan. 1993, doi: 10.1016/0301-5629(93)90003-7.

[54] P. Tortoli, V. Michelassi, G. Bambi, F. Guidi, and D. Righi, "Interaction between secondary velocities, flow pulsation and vessel morphology in the common carotid artery," Ultrasound Med. Biol., vol. 29, no. 3, pp. 407-415, Mar. 2003.

[55] V. L. Newhouse, K. S. Dickerson, D. Cathignol, and J.-Y. Chapelon, "Three-dimensional vector flow estimation using two transducers and spectral width," IEEE Trans. Ultrason. Ferroelectr. Freq. Control, vol. 41, no. 1, pp. 90-95, Jan. 1994, doi: 10.1109/58.265826.

[56] P. Tortoli, G. Bambi, and S. Ricci, "Accurate Doppler angle estimation for vector flow measurements," IEEE Trans. Ultrason. Ferroelectr. Freq. Control, vol. 53, no. 8, pp. 1425-1431, Aug. 2006, doi: 10.1109/TUFFC.2006.1665099.

[57] J. R. Overbeck, K. W. Beach, and D. E. Strandness, "Vector doppler: Accurate measurement of blood velocity in two dimensions," Ultrasound Med. Biol., vol. 18, no. 1, pp. 19-31, Jan. 1992, doi: 10.1016/0301-5629(92)90004$\mathrm{T}$

[58] B. Dunmire, K. W. Beach, K.-H. Labs, M. Plett, and D. E. Strandness Jr., "Cross-beam vector Doppler ultrasound for angle-independent velocity measurements," Ultrasound Med. Biol., vol. 26, no. 8, pp. 1213-1235, Oct. 2000, doi: 10.1016/S0301-5629(00)00287-8.

[59] E. Boni, F. Fool, M. D. Verweij, H. J. Vos, and P. Tortoli, "On the efficacy of in-probe pre-amplifiers for piezoelectric 2D arrays," in 2020 IEEE International Ultrasonics Symposium (IUS), Las Vegas, NV, USA, Sep. 2020, pp. 1-4, doi: 10.1109/IUS46767.2020.9251800.

[60] A. Østvik, E. Smistad, S. A. Aase, B. O. Haugen, and L. Lovstakken, "Real-Time Standard View Classification in Transthoracic Echocardiography Using Convolutional Neural Networks," Ultrasound Med. Biol., vol. 45, no. 2, pp. 374-384, Feb. 2019, doi: 10.1016/j.ultrasmedbio.2018.07.024. 